\title{
A CONGRUENCE FOR PRIMES
}

\author{
ZHI-WEI SUN
}

(Communicated by William Adams)

Abstract. With the help of the Pell sequence we obtain the following new congruence for odd primes:

$$
\sum_{k=1}^{(p-1) / 2} \frac{1}{k \cdot 2^{k}} \equiv \sum_{k=1}^{[3 p / 4]} \frac{(-1)^{k-1}}{k} \quad(\bmod p) .
$$

\section{INTRODUCTION}

Congruences for primes are of great interest. Some examples are

$$
\begin{gathered}
a^{p} \equiv a(\bmod p) \quad(\text { Fermat's little theorem }), \\
(p-1) ! \equiv-1(\bmod p) \quad(\text { Wilson's theorem }), \\
\left(\begin{array}{c}
2 p-1 \\
p
\end{array}\right) \equiv 1\left(\bmod p^{3}\right) \text { for primes } p>3 \quad \text { (Wolstenholme's theorem). }
\end{gathered}
$$

In this paper we shall establish the following congruence for odd primes:

$$
\sum_{k=1}^{(p-1) / 2} \frac{1}{k \cdot 2^{k}} \equiv \sum_{k=1}^{[3 p / 4]} \frac{(-1)^{k-1}}{k}(\bmod p)
$$

where $[\cdot]$ is the greatest integer function.

\section{SOME PREPARATIONS}

Let

$$
\left[\begin{array}{l}
n \\
r
\end{array}\right]=\sum_{\substack{k=0 \\
k \equiv r(\bmod 8)}}^{n}\left(\begin{array}{l}
n \\
k
\end{array}\right)
$$

Using

$$
\left(\begin{array}{l}
n \\
k
\end{array}\right)=\left(\begin{array}{c}
n \\
n-k
\end{array}\right) \text { and }\left(\begin{array}{c}
n+1 \\
k
\end{array}\right)=\left(\begin{array}{l}
n \\
k
\end{array}\right)+\left(\begin{array}{c}
n \\
k-1
\end{array}\right),
$$

one can easily prove that

$$
\left[\begin{array}{l}
n \\
r
\end{array}\right]=\left[\begin{array}{c}
n \\
n-r
\end{array}\right], \quad\left[\begin{array}{c}
n+1 \\
r
\end{array}\right]=\left[\begin{array}{l}
n \\
r
\end{array}\right]+\left[\begin{array}{c}
n \\
r-1
\end{array}\right] .
$$

Received by the editors August 14, 1993.

1991 Mathematics Subject Classification. Primary 11A07, 11A41, 11 B37. 
So we have

$$
\begin{aligned}
{\left[\begin{array}{c}
n+2 \\
2 r
\end{array}\right] } & =\left[\begin{array}{c}
n+1 \\
2 r
\end{array}\right]+\left[\begin{array}{c}
n+1 \\
2 r-1
\end{array}\right]=\left[\begin{array}{c}
n \\
2 r
\end{array}\right]+\left[\begin{array}{c}
n \\
2 r-1
\end{array}\right]+\left[\begin{array}{c}
n \\
2 r-1
\end{array}\right]+\left[\begin{array}{c}
n \\
2 r-2
\end{array}\right] \\
& =\left[\begin{array}{c}
n \\
2 r
\end{array}\right]+2\left[\begin{array}{c}
n \\
2((n+1) / 2-r)
\end{array}\right]+\left[\begin{array}{c}
n \\
2(r-1)
\end{array}\right] .
\end{aligned}
$$

By induction on $n$, one can prove the following result (due to Zhi-Hong Sun [1]) involving the Pell sequence $\left\{P_{n}\right\} \quad\left(P_{0}=0, P_{1}=1, P_{k+1}=2 P_{k}+P_{k-1}\right)$ and its companion $\left\{Q_{n}\right\} \quad\left(Q_{0}=2, Q_{1}=2, Q_{k+1}=2 Q_{k}+Q_{k-1}\right)$.

Lemma 1. Let $n>0$ be odd. We have

(i) if $n \equiv 1(\bmod 8)$, then

$$
\left[\begin{array}{c}
n \\
2 r
\end{array}\right]=2^{n-3}+(-1)^{r} 2^{(n-5) / 2}+(-1)^{[r / 2]+(n-1) / 8} 2^{(n-5) / 4} P_{\left(n+(-1)^{r}\right) / 2} ;
$$

(ii) if $n \equiv 3(\bmod 8)$, then

$$
\left[\begin{array}{c}
n \\
2 r
\end{array}\right]=2^{n-3}-(-1)^{r} 2^{(n-5) / 2}+(-1)^{[r / 2]+(n-3) / 8} 2^{(n-11) / 4} Q_{\left(n-(-1)^{r}\right) / 2}
$$

(iii) if $n \equiv 5(\bmod 8)$, then

$$
\left[\begin{array}{c}
n \\
2 r
\end{array}\right]=2^{n-3}-(-1)^{r} 2^{(n-5) / 2}+(-1)^{[(r+1) / 2]+(n+3) / 8} 2^{(n-5) / 4} P_{(n-(-1) r) / 2}
$$

(iv) if $n \equiv 7(\bmod 8)$, then

$$
\left[\begin{array}{c}
n \\
2 r
\end{array}\right]=2^{n-3}+(-1)^{r} 2^{(n-5) / 2}+(-1)^{[(r+1) / 2]+(n+1) / 8} 2^{(n-11) / 4} Q_{(n+(-1) r) / 2} .
$$

From Lemma 1 it follows that for odd $n>0$

$$
\begin{aligned}
n \sum_{k=1}^{[(n-1) / 4]} \frac{1}{k}\left(\begin{array}{c}
n-1 \\
4 k-1
\end{array}\right) & =4 \sum_{\substack{k=1 \\
4 \mid k}}^{n} \frac{n}{k}\left(\begin{array}{l}
n-1 \\
k-1
\end{array}\right) \\
& =4 \sum_{\substack{k=0 \\
4 \mid k}}^{n}\left(\begin{array}{l}
n \\
k
\end{array}\right)-4=4\left(\left[\begin{array}{l}
n \\
0
\end{array}\right]+\left[\begin{array}{l}
n \\
4
\end{array}\right]\right)-4 \\
& =4 \times\left(2^{n-2}+(-1)^{\left(n^{2}-1\right) / 8} 2^{(n-3) / 2}\right)-4 \\
& =2\left(2^{n-1}-1\right)+2(-1)^{\left(n^{2}-1\right) / 8} \\
& \times\left(2^{(n-1) / 2}-(-1)^{\left(n^{2}-1\right) / 8}\right), \\
\sum_{k=1}^{[(n+1) / 4]} \frac{1}{2 k-1}\left(\begin{array}{c}
n-1 \\
4 k-3
\end{array}\right)= & 2 \sum_{k=0}^{n} \frac{n}{k}\left(\begin{array}{l}
n-1 \\
k-1
\end{array}\right)=2\left(\left[\begin{array}{l}
n \\
2
\end{array}\right]+\left[\begin{array}{l}
n \\
6
\end{array}\right]\right) \\
& 4 \mid k+2 \\
= & 2\left(2^{n-2}-(-1)^{\left(n^{2}-1\right) / 8} 2^{(n-3) / 2}\right) \\
= & 2^{n-1}-1-(-1)^{\left(n^{2}-1\right) / 8}\left(2^{(n-1) / 2}-(-1)^{\left(n^{2}-1\right) / 8}\right) .
\end{aligned}
$$


So we have

Lemma 2. Let $n>0$ be odd. Then

$$
\begin{gathered}
F_{n}+(-1)^{\left(n^{2}-1\right) / 8} E_{n}=\frac{1}{2} \sum_{k=1}^{[(n-1) / 4]} \frac{1}{k}\left(\begin{array}{c}
n-1 \\
4 k-1
\end{array}\right), \\
F_{n}-(-1)^{\left(n^{2}-1\right) / 8} E_{n}=\sum_{k=1}^{[(n+1) / 4]} \frac{1}{2 k-1}\left(\begin{array}{c}
n-1 \\
4 k-3
\end{array}\right)
\end{gathered}
$$

where

$$
F_{n}=\frac{2^{n-1}-1}{n} \text { and } E_{n}=(-1)^{\left(n^{2}-1\right) / 8} \frac{2^{(n-1) / 2}-(-1)^{\left(n^{2}-1\right) / 8}}{n} .
$$

For the sequences $\left\{P_{n}\right\}$ and $\left\{Q_{n}\right\}$, it is well known that

$$
P_{n}=\frac{1}{2 \sqrt{2}}\left[(1+\sqrt{2})^{n}-(1-\sqrt{2})^{n}\right], \quad Q_{n}=(1+\sqrt{2})^{n}+(1-\sqrt{2})^{n} .
$$

From this one can easily derive that

$$
P_{n+1}^{2}+P_{n}^{2}=P_{2 n+1}=\frac{1}{8}\left(Q_{n+1}^{2}+Q_{n}^{2}\right) .
$$

This property will be used below.

Lemma 3. Suppose that $n>0$ is odd. Then

$$
\begin{aligned}
2^{(n-1) / 2} P_{n}=1 & +\frac{n}{2} \sum_{k=1}^{[(n-1) / 4]} \frac{(-1)^{k}}{k}\left(\begin{array}{c}
n-1 \\
4 k-1
\end{array}\right) \\
+\frac{n^{2}}{4} & {\left[\left(\sum_{k=1}^{[(n-1) / 4]} \frac{(-1)^{k}}{2 k}\left(\begin{array}{c}
n-1 \\
4 k-1
\end{array}\right)\right)^{2}\right.} \\
& \left.+\left(\sum_{k=1}^{[(n+1) / 4]} \frac{(-1)^{k-1}}{2 k-1}\left(\begin{array}{c}
n-1 \\
4 k-3
\end{array}\right)\right)^{2}\right] .
\end{aligned}
$$

Proof. By Lemma 1 we have

$$
\begin{aligned}
\left(\left[\begin{array}{c}
n \\
2 r
\end{array}\right]\right. & \left.-2^{n-3}-(-1)^{r+\left(n^{2}-1\right) / 8} 2^{(n-5) / 2}\right)^{2} \\
& +\left(\left[\begin{array}{c}
n \\
2(r+1)
\end{array}\right]-2^{n-3}-(-1)^{r+1+\left(n^{2}-1\right) / 8} 2^{(n-5) / 2}\right)^{2} \\
& = \begin{cases}2^{(n-5) / 2}\left(P_{(n+1) / 2}^{2}+P_{(n-1) / 2}^{2}\right)=2^{(n-5) / 2} P_{n} & \text { if } n \equiv 1(\bmod 4), \\
2^{(n-11) / 2}\left(Q_{(n+1) / 2}^{2}+Q_{(n-1) / 2}^{2}\right)=2^{(n-5) / 2} P_{n} & \text { if } n \equiv 3(\bmod 4) .\end{cases}
\end{aligned}
$$

Putting $r=0$ and noticing that

$$
\left[\begin{array}{l}
n \\
0
\end{array}\right]=1+\sum_{\substack{k=1 \\
8 \mid k}}^{n-1}\left(\begin{array}{l}
n \\
k
\end{array}\right)=1+n C_{n}, \quad\left[\begin{array}{l}
n \\
2
\end{array}\right]=\sum_{\substack{k=1 \\
k \equiv 2(\bmod 8)}}^{n-1}\left(\begin{array}{l}
n \\
k
\end{array}\right)=n \dot{D_{n}}
$$


where

$$
C_{n}=\sum_{k=1}^{[(n-1) / 8]} \frac{1}{8 k}\left(\begin{array}{c}
n-1 \\
8 k-1
\end{array}\right) \text { and } D_{n}=\sum_{k=1}^{[(n+5) / 8]} \frac{1}{8 k-6}\left(\begin{array}{c}
n-1 \\
8 k-7
\end{array}\right)
$$

we then get

$$
\begin{aligned}
2^{(n+3) / 2} P_{n}= & \left(4\left[\begin{array}{l}
n \\
0
\end{array}\right]-2^{n-1}-(-1)^{\left(n^{2}-1\right) / 8} 2^{(n-1) / 2}\right)^{2} \\
& +\left(4\left[\begin{array}{l}
n \\
2
\end{array}\right]-2^{n-1}+(-1)^{\left(n^{2}-1\right) / 8} 2^{(n-1) / 2}\right)^{2} \\
= & {\left[4+4 n C_{n}-\left(1+n F_{n}\right)-(-1)^{\left(n^{2}-1\right) / 8}\left((-1)^{\left(n^{2}-1\right) / 8}+n E_{n}\right)\right]^{2} } \\
& +\left[4 n D_{n}-\left(1+n F_{n}\right)+(-1)^{\left(n^{2}-1\right) / 8}\left((-1)^{\left(n^{2}-1\right) / 8}+n E_{n}\right)\right]^{2} \\
= & {\left[2+n\left(4 C_{n}-F_{n}-(-1)^{\left(n^{2}-1\right) / 8} E_{n}\right)\right]^{2} } \\
& +n^{2}\left[4 D_{n}-F_{n}+(-1)^{\left(n^{2}-1\right) / 8} E_{n}\right]^{2} \\
= & 4+4 n\left(4 C_{n}-F_{n}-(-1)^{\left(n^{2}-1\right) / 8} E_{n}\right) \\
& +n^{2}\left[\left(4 C_{n}-F_{n}-(-1)^{\left(n^{2}-1\right) / 8} E_{n}\right)^{2}\right. \\
& \left.+\left(4 D_{n}-F_{n}+(-1)^{\left(n^{2}-1\right) / 8} E_{n}\right)^{2}\right] .
\end{aligned}
$$

Using Lemma 2 we find that

$$
\begin{aligned}
4 C_{n} & -F_{n}-(-1)^{\left(n^{2}-1\right) / 8} E_{n}=\sum_{k=1}^{[(n-1) / 8]} \frac{1}{2 k}\left(\begin{array}{c}
n-1 \\
8 k-1
\end{array}\right)-\frac{1}{2} \sum_{k=1}^{[(n-1) / 4]} \frac{1}{k}\left(\begin{array}{c}
n-1 \\
4 k-1
\end{array}\right) \\
& \left.=\sum_{\substack{k=1 \\
2 \mid k}}^{[(n-1) / 4]} \frac{1}{k}\left(\begin{array}{c}
n-1 \\
4 k-1
\end{array}\right)-\frac{1}{2} \sum_{\substack{k=1 \\
2 \mid k}}^{[(n-1) / 4]} \frac{1}{k}\left(\begin{array}{c}
n-1 \\
4 k-1
\end{array}\right)+\sum_{\substack{k=1 \\
2 \nmid k}}^{[(n-1) / 4]} \frac{1}{k}\left(\begin{array}{c}
n-1 \\
4 k-1
\end{array}\right)\right] \\
& =\frac{1}{2} \sum_{n}^{[(n-1) / 4]} \frac{(-1)^{k}}{k}\left(\begin{array}{c}
n-1 \\
4 k-1
\end{array}\right), \\
& =4 F_{n}+(-1)^{\left(n^{2}-1\right) / 8} E_{n} \\
& =\sum_{k=1}^{[(n+5) / 8]} \frac{1}{8 k-6}\left(\begin{array}{c}
n-1 \\
8 k-7
\end{array}\right)-\sum_{k=1}^{[(n+1) / 4]} \frac{1}{2 k-1}\left(\begin{array}{c}
n-1 \\
4 k-3
\end{array}\right) \\
= & 2 \sum_{k=1}^{[(n+1) / 4]} \frac{1}{2 k-1}\left(\begin{array}{c}
n-1 \\
4 k-3
\end{array}\right)-\sum_{k=1}^{[(n+1) / 4]} \frac{1}{2 k-1}\left(\begin{array}{c}
n-1 \\
4 k-3
\end{array}\right) \\
= & \sum_{k=1}^{[(n+1) / 4]} \frac{(-1)^{k-1}}{2 k-1}\left(\begin{array}{c}
n-1 \\
4 k-3
\end{array}\right) .
\end{aligned}
$$

This concludes the proof. 
Lemma 4. Let $n>0$ be odd. Then

$$
P_{n}=2^{(n-1) / 2}+2^{(n-3) / 2} n \sum_{k=1}^{(n-1) / 2} \frac{1}{k \cdot 2^{k}}\left(\begin{array}{c}
n-1 \\
2 k-1
\end{array}\right) .
$$

Proof.

$$
\begin{aligned}
P_{n}-2^{(n-1) / 2} & =\frac{1}{2 \sqrt{2}}\left[(1+\sqrt{2})^{n}-(1-\sqrt{2})^{n}\right]-2^{(n-1) / 2} \\
& =\frac{1}{2 \sqrt{2}} \times 2 \sum_{\substack{k=0 \\
2 \nmid k}}^{n}\left(\begin{array}{c}
n \\
n-k
\end{array}\right)(\sqrt{2})^{k}-(\sqrt{2})^{n-1} \\
& =\sum_{i=1}^{(n-1) / 2}\left(\begin{array}{c}
n \\
n+1-2 i
\end{array}\right) 2^{i-1} \\
& =\frac{1}{2} \sum_{i=1}^{(n-1) / 2} \frac{n}{(n+1) / 2-i}\left(\begin{array}{c}
n-1 \\
2((n+1) / 2-i)-1
\end{array}\right) 2^{(n-1) / 2-((n+1) / 2-i)} \\
& =\frac{n}{2} \sum_{k=1}^{(n-1) / 2} \frac{1}{k}\left(\begin{array}{c}
n-1 \\
2 k-1
\end{array}\right) 2^{(n-1) / 2-k} \\
& =2^{(n-3) / 2} n \sum_{k=1}^{(n-1) / 2} \frac{1}{k \cdot 2^{k}}\left(\begin{array}{c}
n-1 \\
2 k-1
\end{array}\right) . \quad
\end{aligned}
$$

\section{The MAIN RESULT}

Theorem. Let $p$ be an odd prime. Then

$$
\sum_{k=1}^{(p-1) / 2} \frac{1}{k \cdot 2^{k}} \equiv \sum_{k=1}^{[3 p / 4]} \frac{(-1)^{k-1}}{k}(\bmod p)
$$

Proof. From Lemmas 3 and 4 it follows that

$$
\begin{aligned}
2^{p-2} \sum_{k=1}^{(p-1) / 2} \frac{1}{k \cdot 2^{k}}\left(\begin{array}{c}
p-1 \\
2 k-1
\end{array}\right)= & \frac{2^{(p-1) / 2} P_{p}-2^{p-1}}{p} \\
= & -\frac{2^{p-1}-1}{p}+\frac{1}{2} \sum_{k=1}^{[(p-1) / 4]} \frac{(-1)^{k}}{k}\left(\begin{array}{c}
p-1 \\
4 k-1
\end{array}\right) \\
& +\frac{p}{4}\left[\left(\sum_{k=1}^{[(p-1) / 4]} \frac{(-1)^{k}}{2 k}\left(\begin{array}{c}
p-1 \\
4 k-1
\end{array}\right)\right)^{2}\right. \\
& \left.+\left(\sum_{k=1}^{[(p+1) / 4]} \frac{(-1)^{k-1}}{2 k-1}\left(\begin{array}{c}
p-1 \\
4 k-3
\end{array}\right)\right)^{2}\right] .
\end{aligned}
$$

Since $p$ is an odd prime, we have

$2^{p-1} \equiv 1 \quad(\bmod p), \quad\left(\begin{array}{c}p-1 \\ n\end{array}\right)=\frac{(p-1)(p-2) \cdots(p-n)}{1 \times 2 \times \cdots \times n} \equiv(-1)^{n} \quad(\bmod p)$, 
and hence

$$
\frac{1}{2} \sum_{k=1}^{(p-1) / 2} \frac{1}{k \cdot 2^{k}}(-1)^{2 k-1} \equiv-\frac{1}{2} \times \frac{2^{p}-2}{p}+\frac{1}{2} \sum_{k=1}^{[(p-1) / 4]} \frac{(-1)^{k}}{k}(-1)^{4 k-1} \quad(\bmod p) .
$$

Thus

$$
\begin{aligned}
\sum_{k=1}^{(p-1) / 2} \frac{1}{k \cdot 2^{k}} \equiv & \frac{\sum_{k=1}^{p-1}\left(\begin{array}{l}
p \\
k
\end{array}\right)}{p}+\sum_{k=1}^{[(p-1) / 4]} \frac{(-1)^{k}}{k} \equiv \sum_{k=1}^{p-1} \frac{1}{k}\left(\begin{array}{l}
p-1 \\
k-1
\end{array}\right) \\
& +\sum_{k=1}^{[(p-1) / 4]} \frac{(-1)^{p-k}}{p-k}(\bmod p) \\
\equiv & \sum_{k=1}^{p-1} \frac{(-1)^{k-1}}{k}+\sum_{k=p-[(p-1) / 4]}^{p-1} \frac{(-1)^{k}}{k} \\
\equiv & \sum_{k=1}^{p-1-[(p-1) / 4]} \frac{(-1)^{k-1}}{k} \equiv \sum_{k=1}^{[3 p / 4]} \frac{(-1)^{k-1}}{k} \quad(\bmod p)
\end{aligned}
$$

This completes the proof.

Remark. The congruence we proved was first conjectured by the author's brother Zhi-Hong Sun in the summer of 1988 . Although he failed to prove the conjecture, he was able to show the congruence

$$
P_{p}-2^{(p-1) / 2} \equiv-2^{(p-3) / 2} p \sum_{k=1}^{(p-1) / 2} \frac{1}{k \cdot 2^{k}} \quad\left(\bmod p^{2}\right)
$$

and confirmed the conjecture in the case $p \equiv 1(\bmod 8)$.

To end this paper we mention another beautiful congruence (for odd primes)

$$
\sum_{k=1}^{(p-1) / 2} \frac{3^{k}}{k} \equiv \sum_{k=1}^{[(p-1) / 6]} \frac{(-1)^{k}}{k} \quad(\bmod p)
$$

It will be published in [2]. For related work the reader can consult [3] and [4].

\section{REFERENCES}

1. Zhi-Hong Sun, Combinatorial sum $\sum_{k=0, k \equiv r(\bmod m)}^{n}\left(\begin{array}{l}n \\ k\end{array}\right)$ and its applications in number theory (II), J. Nanjing Univ. Math. Biquarterly 10 (1993), 105-118.

2. Zhi-Wei Sun, On the combinatorial sum $\sum_{k=0, k \equiv r(\bmod 12)}^{n}\left(\begin{array}{l}n \\ k\end{array}\right)$ and its number-theoretical applications (to appear).

3. Zhi-Hong Sun and Zhi-Wei Sun, Fibonacci numbers and Fermat's last theorem, Acta Arith. 60 (1992), 371-388.

4. A. Granville and Zhi-Wei Sun, Values of Bernoulli polynomials, Pacific J. Math. (to appear).

Department of Mathematics, Nanjing University, Nanjing 210008, People's Republic OF CHINA 